\title{
Progression or Relapse After Hematologic Improvement
}

National Cancer Institute

\section{Source}

National Cancer Institute. Progression or Relapse After Hematologic Improvement. NCI

Thesaurus. Code C123605.

Disease progression or the return of a disease after a period of remission, occurring after a hematologic improvement. 